\title{
Biophysical and cell-based evidence for differential interactions between the death domains of CD95/Fas and FADD
}

\author{
Cell Death and Differentiation (2007) 14, 1717-1719; doi:10.1038/sj.cdd.4402191; published online 29 June 2007
}

Dear Editor,

CD95/Fas belongs to the death receptor family, which is defined by a distinctive cytoplasmic domain known as the death domain (DD). ${ }^{1,2}$ The DD acts as the recruitment site for adaptor proteins whose role is to initiate the various downstream signalling pathways governed by these receptors. The adaptor protein Fas-associated death domain (FADD) is recruited to the CD95/Fas death domain (Fas-DD) immediately following this receptor's activation by its specific ligand, FasL. ${ }^{3}$ FADD, in turn, interacts with procaspase-8/10 forming a high molecular weight multiprotein complex known as the death-inducing signalling complex (DISC). ${ }^{4-6}$ The DISC acts as the site of caspase activation for the initiation of apoptosis. Fas-DD mutations are present in patients with autoimmune lymphoproliferative syndrome (ALPS) or Canale-Smith syndrome whose lymphocytes are resistant to FasL-induced apoptosis. ${ }^{7}$ Mutant CD95/Fas receptors in such cells interfere with the ability of wild-type receptors to signal apoptosis by disruption of the CD95/Fas-FADD interaction. ${ }^{8,9}$ Although the three-dimensional structures of the isolated DDs of CD95/ Fas $^{9}$ and FADD ${ }^{10,11}$ have been solved by nuclear magnetic resonance (NMR) spectroscopy, the exact nature of their interaction remains elusive.

FADD consists of two separate domains, a DD, which interacts with the CD95/Fas receptor via homotypic association, and a second domain, known as a death effector domain. A recent study has indicated that both domains of FADD are necessary for stable interaction with CD95/Fas, ${ }^{6}$ although this appears potentially at odds with the observation of direct DD/DD binding by yeast two-hybrid analysis ${ }^{12}$ and studies in which overexpression of FADD-DD in cell lines has been shown to confer dominant-negative inhibition of CD95/Fas signalling. ${ }^{13}$ We have attempted to address these issues and to develop systems to fully characterize the DD/DD interaction both in vitro and in a biological context.

Initially, we recapitulated yeast two-hybrid experiments that indicate a productive interaction between Fas-DD and the DD of FADD ${ }^{12}$ (Figure 1a). We then created a variety of point mutants of Fas-DD for additional tests. Here, we focus upon three mutants that are further examined by NMR spectroscopy and cell-based assay. D244A is one of the most frequent CD95/Fas mutations found in type la ALPS patients and leads to abrogation of both FasL-induced apoptosis and Fas/FADD interaction. ${ }^{8,9}$ The K247A substitution has been shown to have little or no effect on the CD95/Fas-FADD interaction. ${ }^{9}$ Both of these residues lie on the solvent-exposed face of helix $\alpha 3$ of Fas-DD (Supplementary Figure 1), which is well documented as being a focus for the interaction of CD95/ Fas and FADD. ${ }^{9}$ We also generated the K280A DD mutant. K280 is found on the surface of helix $\alpha 5$ of Fas-DD and has not previously been implicated in interaction with FADD. Yeast two-hybrid tests revealed no evidence for productive interaction of Fas-DD D244A with FADD-DD (Figure 1a), whereas the K247A mutant had no effect on the interaction. The FasDD K280A protein yielded perceptible but low levels of cell growth, suggestive of a weakened interaction. These data were subsequently mimicked by an in vitro surface plasmon resonance study using immobilized wild-type and mutant glutathione S-transferase-Fas-DD (GST-Fas-DD) fusion proteins with FADD-DD as the analyte (Figure 1b).

To fully understand the mechanism of CD95/Fas signal transduction, it is desirable that the methods of threedimensional structural analysis are brought to bear on the composition and organization of the CD95/Fas-dependent DISC and the mechanism of its formation. Attempts to study Fas-DD have been hampered by its well-documented intrinsic tendency to form soluble aggregates at physiological $\mathrm{pH} .{ }^{9} \mathrm{We}$ were able to overcome this difficulty by fusing this DD to the C-terminus of the immunoglobulin-binding B1 domain from streptococcal protein $\mathrm{G}$ (Gb1). Fusion with the 56-residue Gb1 domain has been shown to enhance the tractability of a large number of proteins and has been dubbed a 'solubility enhancement tag'. ${ }^{14}$ Titration of an unlabelled sample of Gb1Fas-DD into a ${ }^{15} \mathrm{~N}$-labelled sample of FADD-DD protein was followed by $\left[{ }^{1} \mathrm{H},{ }^{15} \mathrm{~N}\right]$-heteronuclear single quantum coherence (HSQC) NMR spectroscopy, and this resulted in a monotonic loss of the majority of signals from the FADD-DD polypeptide (Figure 1c). Supplementary Figure 2 shows the plotted intensity of two well-resolved FADD-DD backbone amide group resonances, Glu154 ( $\alpha 4-\alpha 5$ loop) and Ala164 (helix $\alpha 5$ ), as a function of the concentration of added Gb1-Fas-DD. Only cross-peaks from highly mobile regions of the FADD-DD polypeptide, or from a subset of side-chain $\mathrm{NH}_{2}$-groups, remain at the end of the titration. The three mutants of Fas-DD - D244A, K247A, and K280A - were produced as Gb1-FasDD fusion proteins and were found to be suitable for analysis by NMR spectroscopy. 2D $\left[{ }^{1} \mathrm{H},{ }^{15} \mathrm{~N}\right]-\mathrm{HSQC}$ spectra of these three polypeptides indicated that the three substitutions do not disrupt the overall folding characteristics of CD95/Fas-DD (data not shown). Titrations of the three Gb1-Fas-DD mutants with ${ }^{15} \mathrm{~N}-\mathrm{FADD}-\mathrm{DD}$ were performed and monitored using 
a

\begin{tabular}{lll}
\hline $\begin{array}{l}\text { Gal4-AD prey } \\
\text { fusion }\end{array}$ & $\begin{array}{l}\text { Gal4-BD bait } \\
\text { fusion }\end{array}$ & Interaction \\
\hline CD95/Fas-DD & FADD-DD & +++ \\
CD95/Fas-DD & CD95/Fas-DD & - \\
FADD-DD & FADD-DD & - \\
CD95/Fas-DD D244A & FADD-DD & - \\
CD95/Fas-DD K247A & FADD-DD & +++ \\
CD95/Fas-DD K280A & FADD-DD & + \\
\hline
\end{tabular}

b

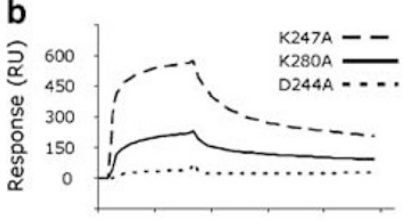

$150 \quad 350 \quad 550 \quad 750 \quad 950$

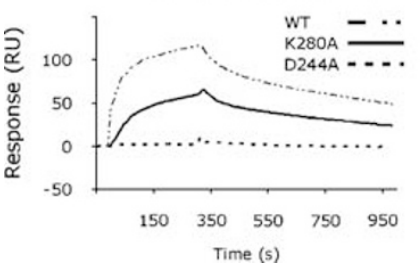

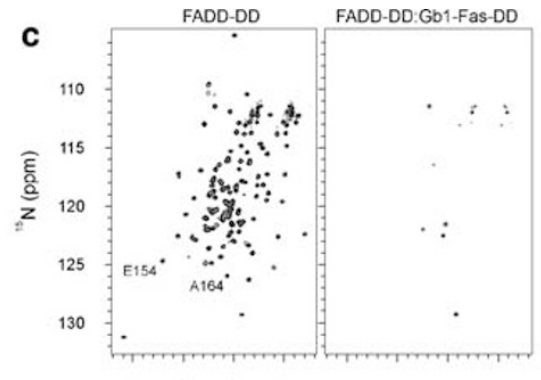

$\begin{array}{llll}10 & 9 & 8 & 7\end{array}$
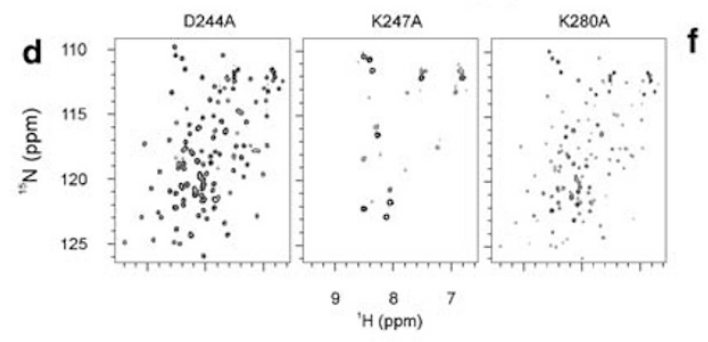

f

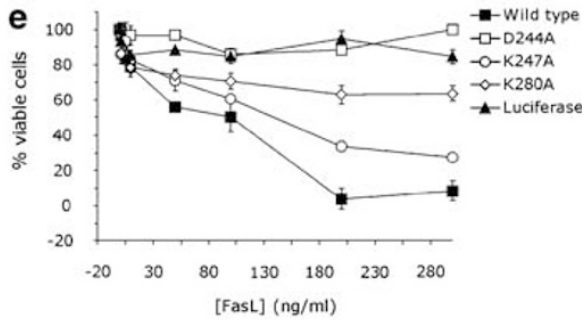

\begin{tabular}{lllll}
\hline \multicolumn{5}{c}{ TUNEL-Positive Cells } \\
\hline & Huh7-Fas & $\begin{array}{l}\text { Huh7-Fas } \\
\text { D244A }\end{array}$ & $\begin{array}{l}\text { Huh7-Fas } \\
\text { K247A }\end{array}$ & $\begin{array}{l}\text { Huh7-Fas } \\
\text { K280A }\end{array}$ \\
\hline untreated & 3 & 5 & 1 & 10 \\
FasL & 318 & 3 & 339 & 173 \\
SSp & 158 & 96 & 106 & 83 \\
-TdT & 0 & ND & ND & ND \\
\hline
\end{tabular}

Figure $1 \mathrm{CD} 95 / \mathrm{Fas}$ and FADD death domains form a stable, soluble complex in vitro. (a) Gal4 activation domain fusions of various constructs were tested for their interaction with Gal4 DNA binding fusion constructs in a yeast two-hybrid assay. Results are displayed as the amount of yeast growth on His-medium scored relative to the wild-type constructs. FADD-DD corresponding to amino-acid residues 93-192 was found to productively interact with Fas-DD (residues 202-307). (b) Binding of FADD-DD to GST-Fas-DD constructs as measured by surface plasmon resonance. FADD-DD analyte was injected over a CM4-sensor surface modified with approximately equimolar amounts of GST-Fas-DD proteins, immobilized by amine coupling. The bulk refractive index change observed in the flow cell similarly modified with unfused GST control was subtracted in each case. (c) $2 \mathrm{D}\left[{ }^{1} \mathrm{H},{ }^{15} \mathrm{~N}\right]-\mathrm{HSQC}$ spectra of $0.4 \mathrm{mM}{ }^{15} \mathrm{~N}$-FADD-DD before and after the addition of a $2 \mathrm{molar}$ excess of unlabelled Gb1-Fas-DD. See Supplementary Figure 2 for a plot of the E154 and A164 cross-peak intensity as a function of the concentration of Gb1-Fas-DD. (d) Effects of CD95/Fas DD mutations on the interaction with FADD-DD. $\left[{ }^{1} \mathrm{H},{ }^{15} \mathrm{~N}\right]-\mathrm{HSQC}$ spectra of $0.4 \mathrm{mM}{ }^{15} \mathrm{~N}-\mathrm{FADD}-\mathrm{DD}$ recorded with the addition of $2 \mathrm{~mol}$ equivalent of Gb1Fas-DD D244A, K247A, and K280A. (e) CD95/Fas K280A mutant attenuates FasL-induced apoptosis. CD95/Fas-expressing cell lines expressing receptor are killed by FasL only if the receptor retains FADD-binding activity. Results represent the percentage of living (actively metabolizing) cells - as measured by MTT assay - after incubation of cell lines with increasing doses of FasL. Cells expressing wild-type CD95/Fas or the K247A mutant died in a dose-dependent manner following exposure to FasL. Cells expressing CD95/Fas-D244A or luciferase were completely resistant to FasL and cells expressing CD95/Fas-K280A died with reduced kinetics relative to cells with wild-type CD95/Fas. Data are presented as mean values $(n=3)$ with error bars indicating standard deviation, representative of two independent experiments. (f) Effect of CD95/Fas mutations on apoptotic cell death determined by TUNEL assay. The number of TUNEL-positive cells was counted from fields of at least 1000 cells chosen randomly for each experiment. The levels of death observed were comparable to those observed by MTT assay. In control experiments, omitting the terminal deoxynucleotide transferase resulted in no TUNEL staining. FasL, CD95/Fas ligand; ssp, staurosporine

heteronuclear NMR spectroscopy. Figure 1d shows spectra of FADD-DD in the presence of 2 mol equivalents of each of the Gb1-Fas-DD variants. For Gb1-Fas-DD K247A, as for the wildtype protein, the majority of the FADD-DD cross-peaks were 'depleted' from the spectrum, providing further evidence that the CD95/Fas K247A substitution has no bearing on the interaction between Fas-DD and FADD-DD. Strikingly, the spectrum of FADD-DD was minimally perturbed in the presence of excess Gb1-Fas-DD D244A: the D244A substitution abrogated the Fas-DD interaction with FADD-DD in the NMR experiment, as would be predicted from in vitro and cellbased observations of CD95/Fas mutants at this residue. ${ }^{8,9}$ The spectrum of FADD-DD in the presence of Gb1-Fas-DD K280A displayed intermediate characteristics: cross-peak intensities were diminished without being reduced to the noise.

These combined data provide convincing evidence for a direct interaction between the DDs of CD95/Fas and FADD that is sensitive to single-residue mutations on the surface of Fas-DD. The global nature of the changes to the NMR spectrum of FADD-DD in the presence of Gb1-Fas-DD indicates that the FADD-DD:Gb1-Fas-DD complex is of such a size that the majority of Fas-DD and FADD-DD amide group cross-peaks have a line width that makes them essentially invisible to NMR, while the tethered Gb1 domain demonstrates free rotation about the connecting peptide linker, along with a small number of highly mobile, mostly chain terminal groups in FADD-DD. Extensive variation of the solution conditions, isotope labelling pattern, and the NMR pulse sequence employed for the measurement demonstrate that the spectroscopic changes are due to slow exchange between free FADDDD and a Fas-DD-bound form of a size that is beyond detection by routine ${ }^{15} \mathrm{~N}$-based NMR spectroscopy. Further work, by ${ }^{13} \mathrm{C}$ NMR and other methods, is underway to establish the size and stoichiometry of the complex formed. Preliminary size exclusion chromatography and analytical ultracentrifugation data have demonstrated the presence of a ca. $160 \mathrm{kDa}$ entity in CD95/Fas-DD:FADD-DD mixtures maintained at concentrations equivalent to the NMR measurements (data not shown). Together, these observations are consistent with a model of DISC formation that implicates a high-order clustering of CD95/ Fas receptors in the cell membrane. ${ }^{15}$

To put our in vitro data in a biological context, a mammalian cell-based assay was developed in which CD95/Fas wild-type and mutant forms were tested for their ability to induce apoptosis in a FasL-stimulated human cell line. The Huh7 cell line chosen for this assay has been described previously as FasL-resistant owing to a lack of CD95/Fas receptor expression, ${ }^{16}$ allowing quantitative experiments without the influence 
of background levels of endogenous CD95/Fas. Moreover, by creating five dox-inducible Huh7 cells lines that stably express wild-type CD95/Fas, one of the three mutant CD95/Fas constructs, or firefly luciferase as a control, we were able to engineer equal levels of expression of CD95/Fas, as detected by Western blotting (Supplementary Figure 3), allowing subsequent quantitative analysis of apoptotic induction upon stimulation with FasL. Importantly, surface expression of CD95/Fas was detectable in all cell lines induced to express the receptor, but not in the cells expressing the luciferase gene (Supplementary Figure 4). These cell lines were tested for their ability to undergo FasL-induced apoptosis by incubation with increasing concentrations of FasL (Figure 1e). The wild-type and K247A constructs were efficient in their ability to transduce FasL death signals as cell cultures expressing these forms of the receptor were almost $100 \%$ dead after $24 \mathrm{~h}$ incubation with $300 \mathrm{ng} / \mathrm{ml}$ FasL. Remarkably, cells expressing the CD95/Fas-D244A construct were entirely resistant to FasL at the concentrations used in these experiments, consistent with data from experiments using $T$ cells taken from patients with type la ALPS that express this mutant. ${ }^{17}$ The cells expressing CD95/Fas K280A exhibited an attenuated response with only about $40 \%$ cell death at the highest FasL concentration. To confirm that the cell death induced in these cell lines by FasL was proceeding via the canonical CD95/Fas apoptotic pathway, two further experiments were carried out. Firstly, it was confirmed that specific inhibition of caspase-8 by the peptide z-IETD-fmk could attenuate the amount of death induced by FasL (Supplementary Figure 5). Secondly, a fluorescent terminal deoxynucleotide transferase-mediated, dUTP nick-end labelling (TUNEL) assay was employed to confirm genomic DNA cleavage during cell death, a strong marker of the apoptotic phenotype. It can be seen by Hoechst staining that cells undergoing apoptosis (either from treatment with FasL or with staurosporine) showed nuclear condensation and fragmentation (Supplementary Figure 6). For semiquantitative analysis of cell death, 1000 Hoechst-stained cells from each experiment were counted and the number of TUNEL-positive cells recorded. The relative level of apoptosis detected by the TUNEL assay in these cell lines correlates well with the amount of cell death determined by the MTT assay (Figure 1f). Significant correlation was noted between the effects of the three CD95/Fas mutations chosen for analysis in the cellbased assay and their effects on FADD-DD binding in the NMR and yeast two-hybrid experiments. In each case, the K247A mutant exhibited similar effects to wild-type CD95/Fas, the D244A mutation essentially knocked out the activity of the wild-type protein, and the K280A mutant showed an intermediate behaviour. The fact that the K280A mutant is shown here to reduce the ability of CD95/Fas to induce apoptosis both corroborates the NMR data and provides functional evidence for this residue, and helix $\alpha 5$, in the CD95/ Fas-DD:FADD-DD binding event. Alongside reports that multiple surfaces of FADD-DD are implicated in the interaction with death receptor $\mathrm{DD},{ }^{18,19}$ prompting models for the homotypic DD-DD interaction that require more than a single surface of the CD95/Fas-DD, , 10,20 our results provide both corroboration for, and a platform for further exploration of, the high molecular weight complex that is apparently formed. Precedent for multimeric DD/DD complexes has been set by the determination of the RAIDD-DD/PIDD-DD complex structure in which seven protomers of RAIDD-DD interact with five of PIDD-DD to form the core of the caspase-2 activating PIDDosome complex. ${ }^{21}$ Such a well-defined complex has not yet been identified for CD95/Fas-DD bound to FADD-DD, and it remains possible that there is no specifically defined size for this entity.

Acknowledgements. Initial aspects of this work were supported by funds from the British Biotechnology and Biological Sciences Research Council (studentship to BJF) and the Ludwig Institute for Cancer Research (AS, PCD). We acknowledge David Vines for assistance in protein expression, Jurg Tschopp for the full-length CD95/Fas receptor CDNA, and Richard Harris and the MRC Biomedical NMR Centre staff for assistance with NMR spectrometers.

\section{BJ Ferguson 1,4,6, D Esposito ${ }^{2,6}$, J Jovanović2 , A Sankar 2 , PC Driscoll ${ }^{*, 1,2}$ and $H$ Mehmet $^{3,5}$}

${ }^{1}$ Department of Biochemistry and Molecular Biology, Institute of Structural Molecular Biology, University College London, Gower Street, London WC1E 6BT, UK;

2 Division of Molecular Structure, National Institute for Medical Research, Medical Research Council, The Ridgeway, Mill Hill, London NW7 1AA, UK and

${ }^{3}$ Faculty of Medicine, Division of Clinical Sciences, Institute of Reproductive and Developmental Biology, Imperial College London, Hammersmith Hospital Campus, Du Cane Road, London W12 0NN, UK

${ }^{4}$ Current address: Department of Pathology, University of Cambridge, Cambridge, UK

${ }^{5}$ Current address: RY80Y-215, Merck Research Laboratories, Rahway, NJ, USA

6 These authors contributed equally to this work

* Corresponding author: PC Driscoll, Division of Molecular Structure, National Institute for Medical Research, The Ridgeway, Mill Hill, London NW7 1AA, UK. Tel: + 44208 8162061; Fax: + 44208 8162734;

E-mail: pdrisco@nimr.mrc.ac.uk

1. Chan KF, Siegel M, Lenardo JM. Immunity 2000; 13: 419-422.

2. Nagata S. Cell 1997; 88: 355-365.

3. Algeciras-Schimnich A et al. Mol Cell Biol 2002; 22: 207-220.

4. Kischkel FC et al. EMBO J 1995; 14: 5579-5588.

5. Muzio M et al. Cell 1996; 85: 817-827.

6. Sandu C et al. Cell Death Differ 2006; 13: 2052-2061.

7. Fisher GH et al. Cell 1995; 81: 935-946.

8. Martin DA et al. Proc Natl Acad Sci USA 1999; 96: 4552-4557.

9. Huang B et al. Nature 1996; 384: 638-641.

10. Berglund $\mathrm{H}$ et al. J Mol Biol 2000; 302: 171-188.

11. Jeong EJ et al. J Biol Chem 1999; 274: 16337-16342.

12. Boldin MP et al. J Biol Chem 1995; 270: 7795-7798.

13. Newton $\mathrm{K}$ et al. EMBO J 1998; 17: 706-718.

14. Zhou P et al. J Biomol NMR 2001; 20: 11-14.

15. Holler $\mathrm{N}$ et al. Mol Cell Biol 2003; 23: 1428-1440.

16. Muller M et al. J Clin Invest 1997; 99: 403-413.

17. Vaishnaw AK et al. J Clin Invest 1999; 103: 355-363

18. Hill JM et al. J Biol Chem 2004; 279: 1474-1481.

19. Thomas LR et al. Cell Death Differ 2006; 13: 160-162.

20. Weber CH, Vincenz C. Trends Biochem Sci 2001; 26: 475-481.

21. Park HH et al. Cell 2007; 128: 533-546.

Supplementary Information accompanies the paper on Cell Death and Differentiation website (http://www.nature.com/cdd) 